\title{
Une méthode pour quantifier les biomasses de résidus de récolte à la surface des sols après la moisson
}

\author{
Pascal Thiebeau* ${ }^{*}$ et Sylvie Recous \\ INRA, UMR 614, Fractionnement des Agro-Ressources et Environnement (FARE), 2 esplanade R.-Garros, 51100 Reims, France
}

\begin{abstract}
Résumé - L'estimation précise des biomasses de résidus de culture présents sur un sol après une récolte est importante vis-à-vis des enjeux agronomiques et environnementaux de la gestion des matières organiques dans les systèmes cultivés. Elle n'est pas aisée dans les parcelles agricoles en raison d'une forte variabilité de leur distribution sur les sols, liée aux opérations de récolte. L'objectif de cette étude est de proposer une méthode de mesure des biomasses de résidus de culture au champ précise, robuste et générique pour une diversité de cultures. Cette méthode repose sur l'utilisation de placettes de prélèvement $(0,5 \times 0,5 \mathrm{~m})$ localisées selon un transect en tenant compte des opérations de récolte (sens de la récolte, largeur de la récolteuse) de façon à capter cette variabilité. Cette méthode a été testée après les récoltes de 2009 à 2011, en région de « grandes cultures », sur des exploitations pratiquant les techniques culturales simplifiées (TCS) et de semis direct (SD), avec les cultures de blé, maïs grain, orge de printemps, pois protéagineux, colza, féverole et tournesol. La comparaison des résultats obtenus avec un ou deux transects montre que la précision des estimations est suffisante en réalisant un seul transect, ce qui diminue significativement le temps de travail. La précision des estimations de biomasse par cette méthode de prélèvement n'est pas affectée par les modes de gestion (TCS versus SD).
\end{abstract}

Mots clés : estimation de la biomasse / mesure au champ / moisson / résidus de cultures

\begin{abstract}
A method to quantify crop residue biomass left on soil surface after harvesting. The accurate quantification of crop residue biomass left on the soil after harvesting is important, due to the agricultural and environmental issues associated with the management of soil organic matter in croplands. This quantification is difficult in farmers' fields due to a high variability of crop residue distribution on soil surface, because of harvesting operations. The objective of this study was to provide, for measuring crop residue biomass in the field, an accurate, robust and generic method for a large range of crops. This method relies on the use of sampling microplots $(0.5 \times 0.5 \mathrm{~m})$, located along a transect and taking into account harvesting operations (harvesting direction and width) to capture this variability. This method was tested from 2009 to 2011, in farmers' plots after harvesting, on wheat, grain maize, spring barley, field pea, rapeseed, fababean and sunflower crops. The comparison of results obtained with one or two transects (one perpendicular and the other diagonally across harvesting direction) shows that the accuracy of the estimates is sufficient using a single transect, allowing for a reduction of the working time. The accuracy of this method of collection is not affected by soil management practices (shallow till versus no-till).
\end{abstract}

Keywords: biomass measurement / crop harvest / crop residues / field method

\section{Introduction}

L'atténuation du réchauffement climatique peut faire appel à un accroissement du stock de carbone organique des sols (MAAF, 2015) par une modification des pratiques culturales et un accroissement des restitutions aux sols des résidus végétaux après récolte. Dans ce contexte, la réduction ou la

\footnotetext{
* Auteur de correspondance : thiebeau@reims.inra.fr
}

suppression du travail du sol sont des solutions préconisées pour favoriser le stockage de carbone dans les sols agricoles (Arrouays et al., 2002 ; Brunnert, 1996 ; Seguin et Soussana, 2006). Dans ces situations, les résidus de cultures sont maintenus à la surface du sol dans le cas du semis direct (SD) ou partiellement incorporés dans les premiers centimètres de sol en systèmes de techniques culturales simplifiées (TCS). La connaissance des biomasses végétales recyclées est une donnée d'entrée très importante des modèles de simulation des effets des modes d'occupation des sols et des pratiques 
agricoles sur l'évolution des stocks de carbone, mais aussi des modèles d'aide à la décision (ex. modèle de bilan humique ; Andriulo et al., 1999). Ces entrées initiales de carbone sont déterminantes dans le renouvellement des matières organiques du sol (Neto et al., 2010), mais aussi pour la fourniture des nutriments, notamment de l'azote (Maltas et al., 2009). Leur répartition au sol peut être très hétérogène, selon les techniques mises en œuvre à la récolte et les conditions climatiques plus ou moins favorables à leur dispersion lors de la récolte, rendant difficile une estimation précise de leur quantité. L'estimation de ces quantités de résidus peut être envisagée de plusieurs façons. Dans le cadre de dispositifs expérimentaux, Oorst et al. (2007), Pascault et al. (2010) et Ringselle et al. (2014) ont utilisé des placettes délimitées par des cadres de $0,5 \mathrm{~m} \times 0,5 \mathrm{~m}$ pour estimer la masse des résidus végétaux présents sur leurs dispositifs, en réalisant soit un transect perpendiculaire au sens du labour ou du travail du sol, soit des prélèvements aléatoires. Pour être représentatif de la culture étudiée, il suffit alors de moduler le nombre de placettes de prélèvement. Ainsi, Sinclair et Muchow (1999) estiment qu'une surface de prélèvement totale de $1 \mathrm{~m}^{2}$, prise en différents endroits d'une parcelle, est nécessaire pour estimer convenablement la biomasse présente d'une culture. Dans les situations évoquées ci-dessus, la surface totale de prélèvements a oscillé entre 3 et $6 \mathrm{~m}^{2}$, sur des parcelles expérimentales où les interventions culturales étaient maîtrisées par les expérimentateurs. Sur des parcelles d'agriculteurs, l'estimation précise des résidus est plus difficile en raison de l'application de techniques et de largeurs d'outils pouvant être très différentes selon les exploitations.

L'objectif de ce travail est de mettre au point une méthode d'estimation quantitative des résidus de cultures après la moisson, sur des situations agricoles contrastées. Cette méthode doit permettre de réaliser une estimation fiable de la quantité de résidus de récolte, tout en restant une méthode simple et rapide à mettre en œuvre, notamment par une personne seule.

\section{Matériels et méthodes}

Le travail est réalisé sur des exploitations de «grandes cultures » du Nord-Est de la France, où les espèces sont cultivées en culture pure. Les sept exploitations support de notre étude, réparties sur les départements de l'Aube (10) et de la Meuse (55), pratiquaient les TCS ou le SD depuis cinq à dix ans en 2009. Les cultures support de ce travail sont le blé, le maïs grain, l'orge de printemps, l'escourgeon, le pois protéagineux, le colza, la féverole et le tournesol, de 2009 à 2011. Ce choix de cultures nous permet d'avoir des quantités de résidus très différentes et de faire varier les conditions climatiques qui peuvent être contrastées lors des récoltes.

L'estimation des quantités de biomasses de résidus est déterminée dès la récolte des cultures. L'hétérogénéité de leur répartition au sol a été forte en 2009 et 2010 en raison de conditions pluviométriques et venteuses défavorables tout au long de la moisson, rendant plus difficile le broyage des pailles et accentuant la difficulté d'une répartition homogène par les dispositifs de broyage et de dispersion équipant les moissonneuses. Ces mauvaises conditions de récoltes ont été, en fait, une opportunité pour mettre au point un protocole robuste qui sera, a fortiori, applicable en bonnes conditions de récolte.

\subsection{Positionnement des placettes de prélèvement à l'intérieur d'une parcelle}

La méthode d'estimation repose sur :

- une série de prélèvements de résidus sur une largeur de coupe de la moissonneuse, afin d'intégrer un transect de répartition par le broyeur de la moissonneuse, comme ont pu le faire Oorst et al. (2007) et Pascault et al. (2010) ;

- une série de prélèvements en diagonale par rapport à ce transect, afin de prendre en compte la variabilité de répartition des résidus créée en cours de progression de la moissonneuse.

L'unité de surface de prélèvement est constituée d'un cadre de dimensions intérieures de $0,5 \mathrm{~m} \times 0,5 \mathrm{~m}$. Les prélèvements sont réalisés sur des surfaces délimitées par plusieurs cadres constituant des placettes de prélèvement, dont le nombre est fonction de la largeur de coupe de la moissonneuse utilisée sur chaque exploitation. Le test de différentes configurations d'espacements entre les cadres de prélèvement nous a permis de retenir la règle d'une placette de prélèvement par mètre linéaire de fauche, soit, dans nos conditions de travail :

- cinq placettes pour 4,8 à 5,5 m de largeur de coupe ;

- six placettes pour 5,8 à $6,5 \mathrm{~m}$ de largeur de coupe ;

- sept placettes pour 6,8 à 7,5 $\mathrm{m}$ de largeur de coupe.

Nous ajustons ensuite la distance séparant chacun des cadres afin qu'elle soit identique. Chaque estimation de biomasse de résidus résulte donc de la moyenne de cinq à sept placettes, soit 1,25 à $1,75 \mathrm{~m}^{2}$ par transect. Celles-ci sont présentées avec une estimation de l'erreur associée, standardisée (SE), afin de pouvoir être comparées.

Pour réaliser l'estimation au champ, on se place à $50 \mathrm{~m}$ au minimum à l'intérieur des bordures du champ, ce qui élimine les zones de superpositions de pailles qui peuvent exister lorsque la moissonneuse réalise ses demi-tours. Ensuite, on identifie précisément une largeur de coupe de la machine grâce aux hauteurs d'éteules (chaumes) différentes entre deux passages. C'est à partir de ce repérage que l'on réalise le transect de prélèvement linéaire (Fig. 1A), perpendiculaire au sens de récolte. Enfin, c'est à partir de ce transect de prélèvement que l'on réalise le deuxième transect, en diagonale et indépendant du premier, en procédant de la sorte :

- on déplace une placette d'extrémité d'un mètre par rapport à sa base, en respectant le sens de progression de la moissonneuse : cette nouvelle position devient la base de référence (Fig. 1B);

- puis on déplace l'autre placette d'extrémité d'une distance correspondant à une largeur de fauche de la moissonneuse par rapport à la nouvelle base de référence, en respectant également le cheminement de récolte de la moissonneuse (Fig. 1C) ;

- enfin, à l'aide d'un décamètre, on trace la diagonale qui passe par les angles extérieurs des cadres de chaque extrémité, puis on déporte chaque cadre de la ligne de prélèvement latérale contre celle de la diagonale ainsi tracée, tout en respectant la progression de la moissonneuse (Fig. 1D).

Nous avons testé l'hypothèse selon laquelle l'estimation obtenue est répétable sur un même champ. Pour cela, nous avons réalisé une seconde estimation (répétition 2, notée R2) 

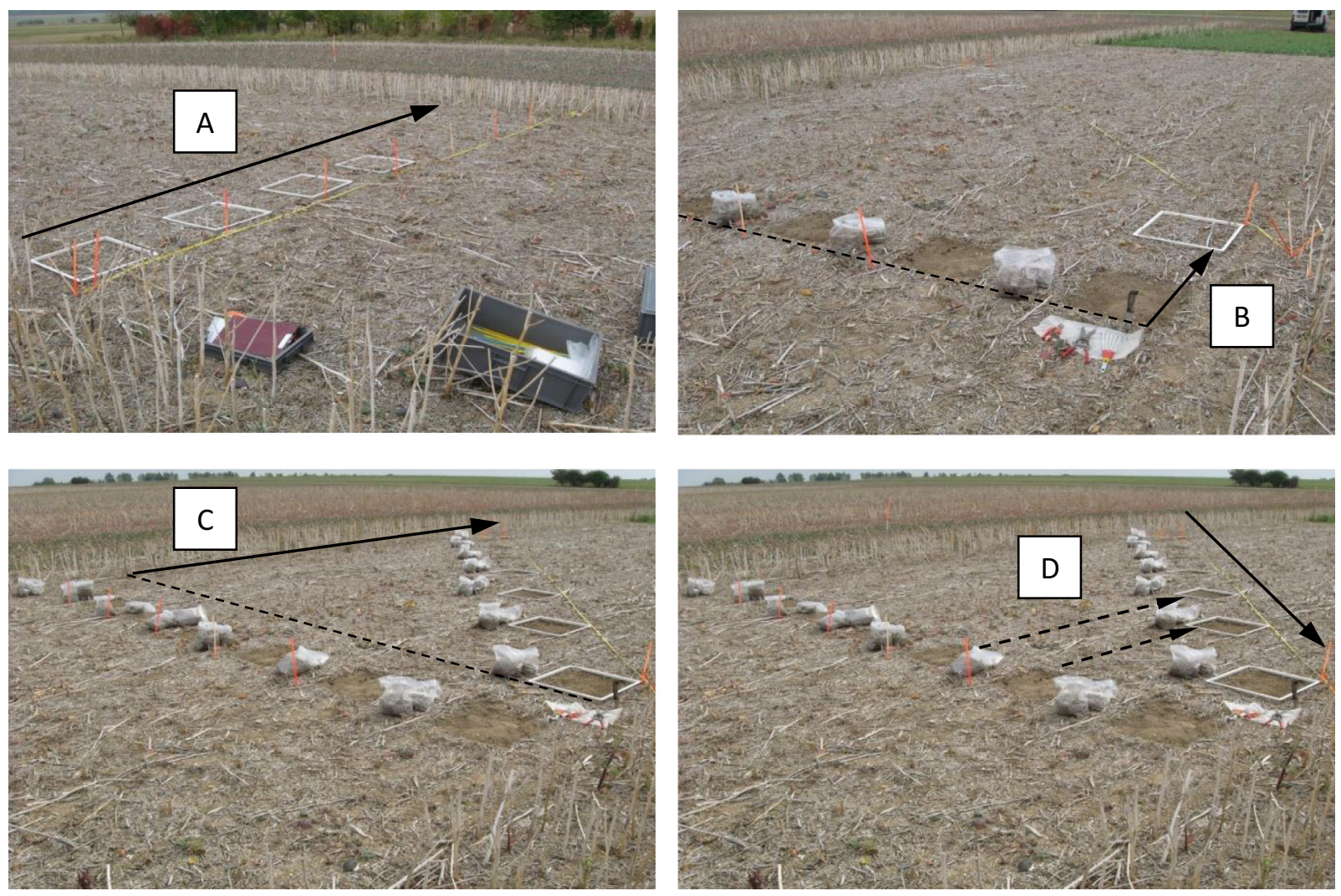

Fig. 1. Méthodologie d'estimation des biomasses de résidus au champ. A : réalisation du transect perpendiculaire ; B-D : trois étapes de réalisation du transect en diagonale.

Fig. 1. Methodology of estimation of crop residues in the field. A: microplots along a perpendicular transect; B-D: three stages of design of the diagonal transect and microplots placement.

en nous éloignant de $50 \mathrm{~m}$ au moins du premier lieu de prélèvement (répétition 1, notée R1).

\subsection{Prélèvements et traitements des échantillons}

Sur chaque placette délimitée par un cadre, nous distinguons un échantillon $\mathrm{A}$ et un échantillon $\mathrm{B}$ :

- l'échantillon A est constitué du paillis "propre », correspondant aux résidus de récolte que l'on peut ramasser à la surface du sol sans incorporer de terre. Cet échantillon comprend les éteules, coupées au ras de la surface du sol ;

- l'échantillon B est constitué des particules de paillis en mélange avec du sol. Il s'agit, dans les situations explorées, de la menue paille (glumes et glumelles des épis) ainsi que des fractions de pailles très broyées : cet échantillon est collecté avec une griffe à six dents souples, qui respecte la rugosité de surface du sol (Currence et Lovely, 1970). Les rares adventices ou plantes de couverture présentes sont exclues de l'échantillon collecté.

Chaque échantillon collecté est placé en poche individuelle pour être traité au laboratoire dans sa totalité.

L'échantillon A est placé directement en étuve ventilée pour être séché durant 48 heures à $80^{\circ} \mathrm{C}$, puis pesé en sortie d'étuve pour l'estimation de sa masse sèche.
L'échantillon B subit une immersion dans un récipient d'eau afin de séparer le sol, qui sédimente, alors que les particules de paillis flottent à la surface de l'eau. Les particules de paillis sont récupérées par filtration à l'aide d'un tamis dont la maille est de $2 \mathrm{~mm}$. Après un pressage manuel pour extraire l'eau de gravité, cette biomasse est séchée en étuve ventilée durant 96 heures à $80^{\circ} \mathrm{C}$, puis pesée pour l'estimation de sa masse sèche.

Le traitement des échantillons A et B permet de connaître la matière sèche totale (MST) de paillis présente à la surface du sol pour chaque placette de mesure.

\subsection{Traitements statistiques des données}

Toutes les données moyennes présentées sont accompagnées de leur erreur d'estimation standardisée, afin de pouvoir être comparées entre elles.

Les analyses de variances sont réalisées à l'aide du programme statistique de Sigma-Plot 12 (Systat Software, 2010) après validation du test de normalité (Shapiro-Wilk). Le classement en groupes homogènes est réalisé en employant le test de Newman et Keuls. Le niveau de signification retenu est $p<0,05$. Les critères d'évaluation des ajustements linéaires sont l'erreur quadratique au modèle (RMSE) et le coefficient de détermination $\left(R^{2}\right)$. 


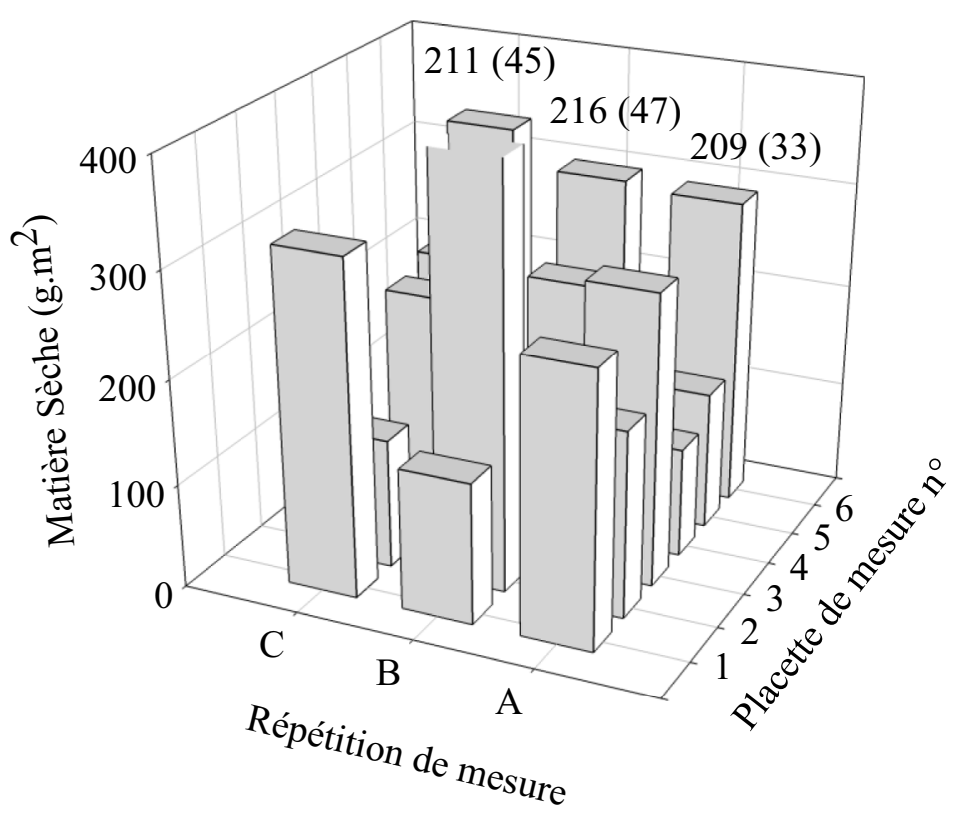

Fig. 2. Exemple de variabilité spatiale observée sur un transect de la moissonneuse après une récolte de pois.

Fig. 2. Example of the spatial variability of pea residues collected in six microplots along a transect perpendicular to the direction of harvest, with three replicated transects.

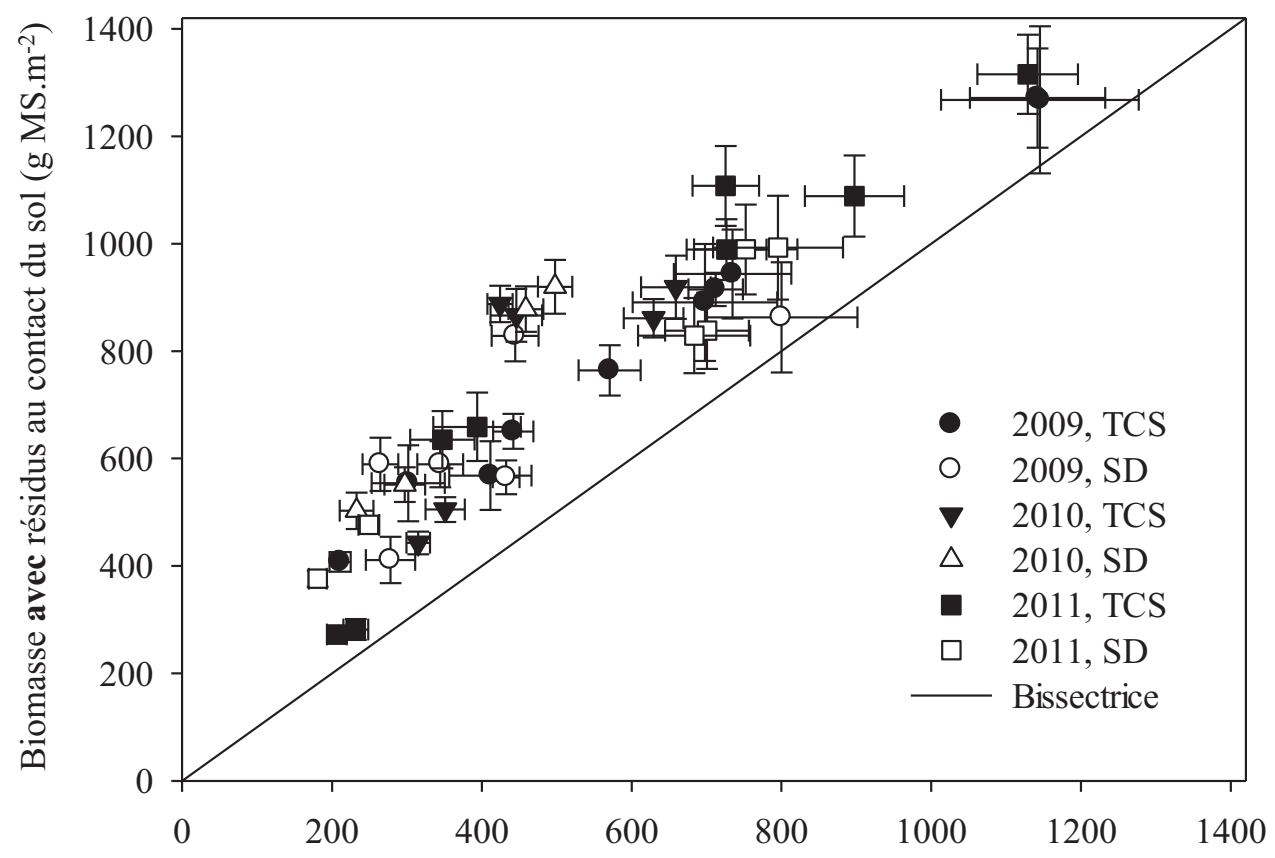

Fig. 3. Comparaison des biomasses estimées avec et sans prise en compte des particules de résidus au contact du sol.

Fig. 3. Comparison of the crop residue dry matters with and without taking into account the residue fraction in contact with the soil.

Biomasse sans les résidus au contact du sol $\left(\mathrm{g} \mathrm{MS} . \mathrm{m}^{-2}\right)$

\section{Résultats}

\subsection{Variabilité spatiale de la biomasse de résidus et effet des pratiques de cultures sur la biomasse mesurée}

Au cours des trois campagnes culturales, nous avons récolté à plusieurs reprises les mêmes espèces, dans des situations culturales proches (TCS ou SD). Nous observons une forte variabilité de la répartition des pailles au sol, derrière la moissonneuse, comme le montrent par exemple les données avec les résidus de pois (Fig. 2). Les mesures réalisées selon le protocole présenté, répétées trois fois de façon indépendante, montrent que les quantités de matière sèche (MS) mesurées sur chaque placette de prélèvement peuvent être très variables, tandis que la moyenne des six placettes $\left(1,5 \mathrm{~m}^{2}\right)$ constituant un transect de chaque répétition n'est pas différente : $209 \pm 33$, $216 \pm 47,211 \pm 45 \mathrm{~g} \mathrm{MS} / \mathrm{m}^{2}$.

La prise en compte des résidus en contact avec le sol (échantillon B) évite de sous-estimer significativement la quantité totale de résidus présents au sol (Fig. 3). L'ajustement 


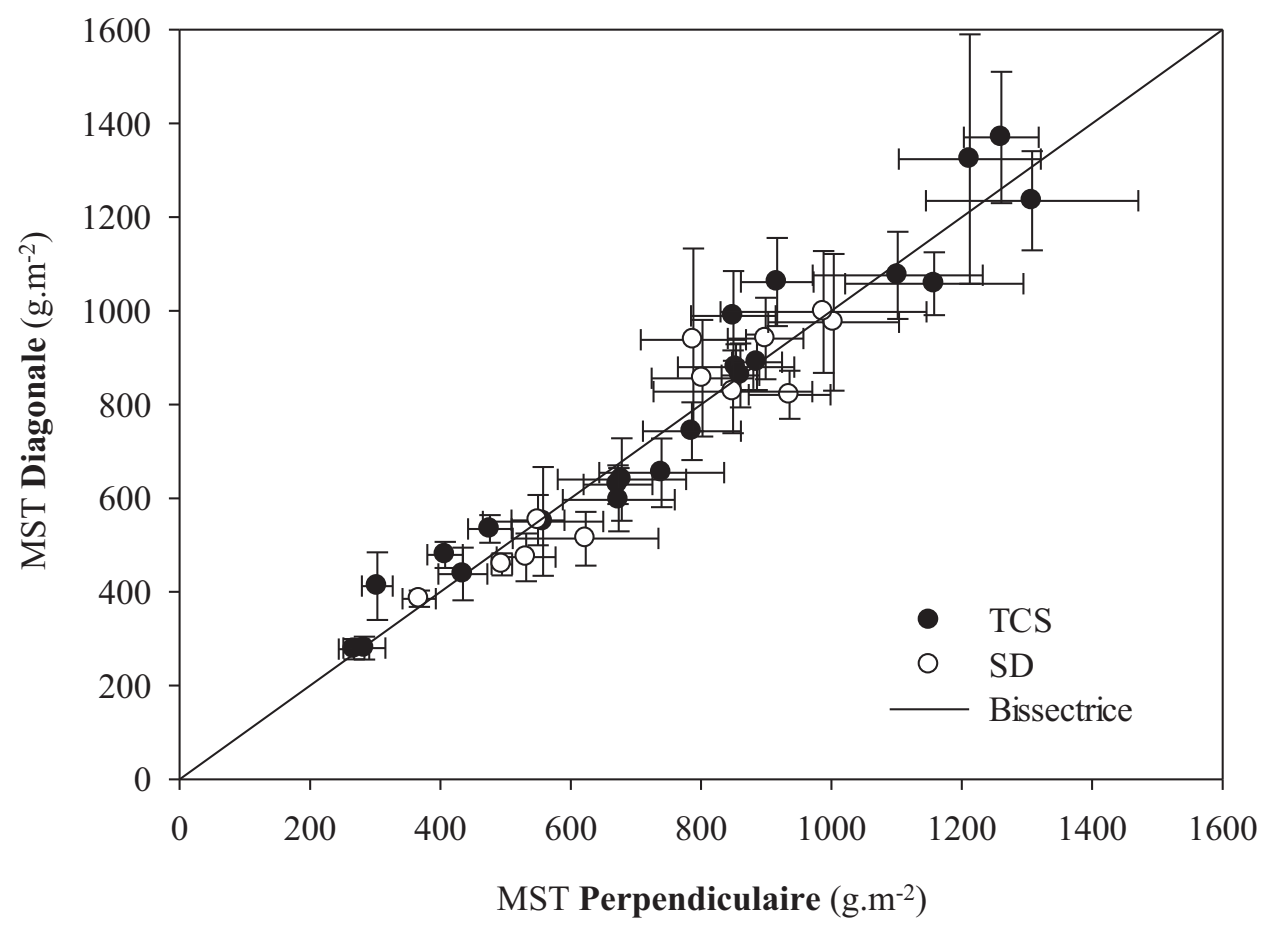

Fig. 4. Comparaison entre les matières sèches de résidus de récolte mesurée le long d'un transect réalisé perpendiculairement au trajet de la moissonneuse et celui réalisé diagonalement au sens de progression de la moissonneuse.

Fig. 4. Comparison of the crop residue dry matters collected along transects realized perpendicularly or diagonally to the direction of harvesting.

linéaire passant par ce nuage de point donne une pente de 0,94 et une constante de $255 \mathrm{~g}$ de MS, avec RMSE $=99 \mathrm{~g}$ MS et $R^{2}=0,8612(p<0,001)$. Cet ajustement est presque parallèle à la première bissectrice. En forçant la pente à celle de la bissectrice (valeur de 1), la valeur de la constante est alors de $225 \mathrm{~g}$ MS, avec RMSE $=100 \mathrm{~g}$ MS et $R^{2}=0,8583(p<0,001)$, ce qui dégrade peu la qualité de l'estimation obtenue précédemment. Dans cette hypothèse, la part de l'échantillon $\mathrm{B}$ représente près de $30 \%$ de la MST de résidus au lieu de $34 \%$ précédemment.

Par ailleurs, l'analyse des pratiques culturales mises en œuvre par chaque agriculteur (TCS ou SD) pour les trois campagnes culturales 2009, 2010 et 2011 ne permet pas de distinguer un effet année $(p=0,91)$, un effet des pratiques culturales $(p=0,36)$ ou une interaction année $\times$ pratique culturale $(p=0,75)$. Nous pouvons donc analyser les données collectées indépendamment des années culturales et des techniques mises en œuvre sur les exploitations support de ce travail. Cependant, nous maintenons une présentation des résultats faisant la distinction entre TCS et SD.

\subsection{Robustesse de la méthode de prélèvement}

Nous avons comparé les estimations quantitatives de résidus réalisées perpendiculairement au sens de la moisson, à celles réalisées en diagonale à la direction de récolte. Les données montrent (Fig. 4) que l'ensemble des valeurs se superposent à la première bissectrice. Leur ajustement linéaire offre une pente très proche de $1(0,997)$ avec une constante de $8 \mathrm{~g}$ MS $\left(\mathrm{RMSE}=72 \mathrm{~g} \mathrm{MS}, R^{2}=0,9370, p<0,001\right)$. En forçant la constante pour qu'elle soit nulle, la pente calculée est de
$1,006\left(\mathrm{RMSE}=72 \mathrm{~g} \mathrm{MS}, R^{2}=0,9369, p<0,001\right)$. L'analyse de variance ne détecte aucun effet année $(p=0,243)$, effet sens de collecte des résidus, perpendiculaire versus diagonale $(p=0,953)$, ou interaction entre ces deux facteurs $(p=0,980)$.

L'estimation de la répétabilité des mesures de MST de résidus, sur un même champ, par la réalisation d'une seconde mesure à $50 \mathrm{~m}$ d'intervalle, que ce soit par un transect perpendiculaire (Fig. 5A) ou un transect en diagonale (Fig. 5B) montre que les données se trouvent sur, ou très proches, de la première bissectrice. Avec le transect perpendiculaire, la RMSE est de $97 \mathrm{~g}$ MS pour un $R^{2}$ supérieur à 0,89 ; tandis qu'avec le transect en diagonale, la RMSE est de $-26 \mathrm{~g} \mathrm{MS}$ pour un $R^{2}$ supérieur à 0,90 . Ces deux ajustements sont statistiquement hautement significatifs. Les résultats de l'analyse de variance ne montrent pas d'effet de l'estimation perpendiculaire $(p=0,808)$, ni d'effet répétition $(p=0,979)$, ni d'interaction mesure perpendiculaire $\times$ répétitions $(p=0,814)$. De même, nous ne décelons pas d'effet des mesures en diagonale $(p=0,589)$, ni d'effet répétitions $(p=0,996)$, ni d'interaction mesure en diagonale $\times$ répétitions $(p=0,740)$.

La prise en compte pour chaque emplacement d'estimation de la MST, d'un transect perpendiculaire et d'un transect en diagonale, caractérisant ainsi un double transect, permet d'améliorer l'estimation de MST initialement obtenue par l'un ou l'autre des transects. La répétition à $50 \mathrm{~m}$ d'intervalle (Fig. 5C) montre que l'on obtient une pente d'ajustement qui se superpose pratiquement à la première bissectrice, avec une constante de $-4 \mathrm{~g}$ de MS. En forçant l'ajustement par l'origine afin d'annuler la constante, on ne détériore ni la $\mathrm{RMSE}=81 \mathrm{~g}$ MS, ni le $R^{2}>0,93$ qui reste hautement significatif $(p<0,001)$. On note l'absence d'effet système de culture 


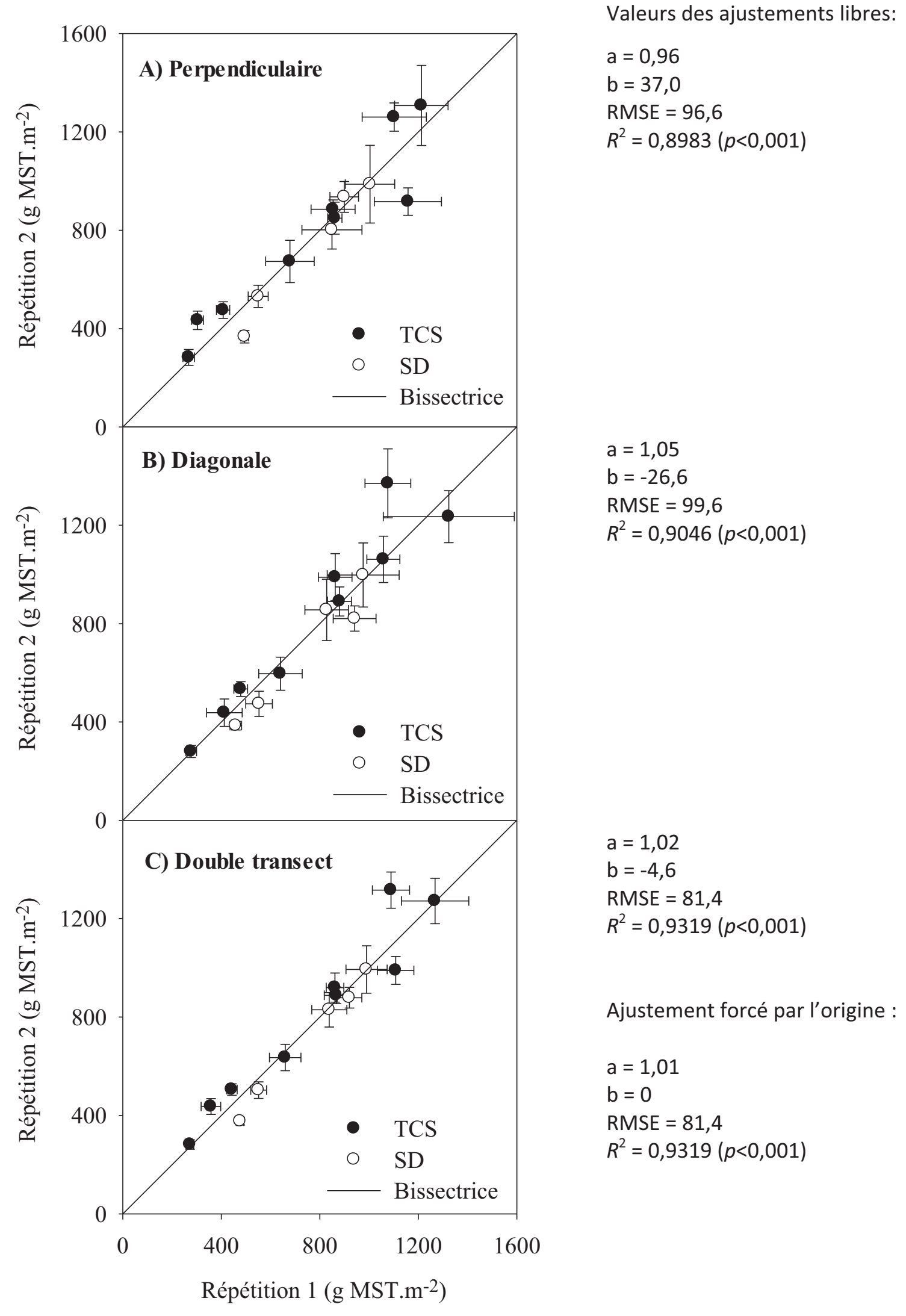

Fig. 5. Tests de robustesse de la méthode en comparant deux répétitions de transect réalisés à $50 \mathrm{~m}$ d'intervalle. A : pour la méthode de transect perpendiculaire ; B : pour la méthode de transect en diagonale ; C : pour la méthode utilisant le double transect.

Fig. 5. Tests of robustness of the method, by comparing two transect replicates performed at 50-m interval. A: for the perpendicular transect method; B: for the diagonal transect method; $C$ : for the combined transect method. 
( $p=0,693)$, d'effet répétitions $(p=0,987)$, et d'interaction système de culture $\times$ répétitions $(p=0,775)$.

\section{Discussion}

Au cours des trois années d'expérimentation, les données obtenues ont montré que les cultures laissaient de $267 \pm 23$ à $1370 \pm 140 \mathrm{~g} \mathrm{MS} / \mathrm{m}^{2}$ sur le sol, ce qui montre une forte variabilité des biomasses de résidus (soit 2,7 à 13,7 t/ha) à la récolte. La méthode proposée a bien capté l'hétérogénéité de répartition des résidus de récolte qui existe notamment en conditions de récolte défavorables. Si l'on retient une concentration moyenne en carbone (C) des tissus végétaux de $0,45 \mathrm{~g} \mathrm{C} / \mathrm{g}$ de biomasse, cela représente une quantité de carbone restitué au sol de $1,2 \pm 0,1$ à $6,2 \pm 0,6 \mathrm{tC} /$ ha dans les conditions expérimentales explorées, avec une erreur relative d'estimation de 8 à $10 \%$. Cette incertitude est faible si on la compare aux stocks de carbone des sols cultivés français, qui sont en moyenne de $30 \mathrm{tC} / \mathrm{ha}$ dans la couche $0-20 \mathrm{~cm}$ (Ademe, 2014 ; Ferchaud et al., 2015), et représente alors 0,3 à $2,0 \%$ d'erreur relative.

En situation de non-labour, la majeure partie du paillis va se maintenir plusieurs mois au sol, ce qui aura une action sur les cycles du carbone (Neto et al., 2010), des éléments nutritifs (Naudin et al., 2011) et la dynamique de l'eau dans ces sols (Iqbal et al., 2015). Compte tenu de la sensibilité des modèles de carbone (C) des sols à la connaissance et la précision de ces quantités de résidus, cette méthode d'estimation permet d'améliorer la qualité des prédictions relatives aux modes de gestion ou d'utilisation des terres sur les bilans de carbone des sols.

Cette méthode a été conçue pour être mise en application par une personne seule. Elle montre que l'on obtient une bonne précision de l'estimation de la biomasse végétale restant au sol au champ, après une récolte, quel que soit le sens du transect : perpendiculaire au sens de la récolte ou en diagonale par rapport au sens de la récolte. Il est important de noter que, grâce à l'organisation spatiale de la collecte des échantillons, cette méthode peut être appliquée en un seul lieu du champ, sans répétition. La réalisation d'un double transect, constitué alors de 10 à 14 placettes pour nos situations $\left(2,25-3,25 \mathrm{~m}^{2}\right)$, affine la qualité d'estimation. Le double transect peut être envisagé dans les situations qui nécessitent moins de cinq placettes de prélèvement par transect, ce qui est le cas de petites exploitations familiales (matériel de récolte moins large, mieux adapté au parcellaire). En effet, réaliser un double transect de 10 à 14 placettes, seul, occupe deux à trois heures par situation. Le choix est donc à apprécier selon chaque opérateur.

Lors de la récolte des échantillons au champ, il est nécessaire de collecter la fraction de menue paille en contact avec le sol. En effet, nous avons constaté que cette fraction associée à la terre fine de surface représentait en moyenne près d'un tiers de la fraction totale. Cette fraction varie beaucoup d'un champ à un autre et d'une culture à une autre. Nous préconisons de réaliser cette mesure (échantillons A et $\mathrm{B}$ ) pour toutes les situations étudiées.

Cette méthode d'estimation des résidus, aussi précise soitelle, prend tout son sens sur une unité de sol homogène. Il est évident que le changement de nature de sol au sein d'une même parcelle, qui aurait pour incidence de modifier significative- ment la production de biomasse de la culture, impliquerait de réaliser une estimation de la biomasse au sein de cette zone. L'estimation de la quantité de résidus sur l'ensemble du champ sera donc à établir au prorata de la part qu'occupe chacune des natures de sols.

\section{Conclusion}

Ce travail permet de proposer une méthode standardisée et précise d'estimation de résidus de cultures présents sur le sol après une récolte. Cette estimation est réalisée à partir d'un ensemble de placettes positionnées sur un transect linéaire, réalisé perpendiculairement au sens de la récolte, ou diagonalement au sens de progression de la machine de récolte. La réalisation d'un double transect, intégrant les deux sens de prélèvement, augmente la précision d'estimation, mais se révèle également plus consommatrice de temps de travail. Vis-à-vis des objectifs d'adaptation des pratiques agricoles pour favoriser le stockage de carbone dans les sols, cette méthode, bien qu'étant " précise », permet de fournir une erreur d'estimation aux bilans réalisés. Enfin, cette méthode peut être appliquée à d'autres situations de culture, pour estimer des biomasses prairiales basses sur pied, des biomasses forestières au sol sous futaie, et/ou des biomasses pré-enfouies, en intégrant l'épaisseur de sol d'enfouissement (déchaumage).

Remerciements. Les auteurs remercient chaleureusement Monsieur Denis, de la coopérative Vivescia (Aube) pour ses conseils dans le choix des exploitations, mais aussi Messieurs Acker, Ferté, Gubelin, Lemey et Martens, exploitants agricoles qui ont accepté de mettre à notre disposition un peu de leur temps, et leurs champs, sans contrepartie, afin que nous puissions conduire cette étude. Nous remercions également A. Iqbal pour son aide lors de la collecte des échantillons au cours de l'été 2009. Enfin, nous remercions l'Agence nationale de la recherche (ANR) pour le soutien financier au projet PEPITES ANR-08-STRA-10 (Processus écologiques et processus d'innovations technique et social en agriculture de conservation).

\section{Références}

Ademe. 2014. Carbone organique des sols. L'énergie de l'agroécologie, une solution pour le climat, In: Eglin T, coord., Angers: Ademe Editions, 28p., ISBN: 978-2-358-38-447-6.

Andriulo A, Guerif J, Mary B. 1999. Modelling soil carbon dynamics with various cropping sequences on the rolling pampas. Agronomie 19(5): 365-377.

Arrouays D, Balesdents J, Germon J-C, Jayet P-A, Soussana J-F. 2002. Contribution à la lutte contre l'effet de serre. Stocker du carbone dans les sols agricoles en France ? Expertise scientifique collective, Synthèse, INRA, $32 \mathrm{p}$.

Brunnert H. 1996. The significance of agriculture and forestry for the global carbon cycle. How can they contribute to sequester increasing amounts of $\mathrm{CO}_{2}$ in the atmosphere. Berichte Uber Landwirtschaft 74(1): 44-65.

Currence HD, Lovely WG. 1970. The analysis of soil surface roughness. Trans. ASAE 13(6): 710-714.

Ferchaud F, Vitte G, Mary B. 2015. Changes in soil carbon stocks under perennial and annual bioenergy crops. Glob. Chang. Biol. Bioenergy 8: 290-306. 
Iqbal A, Aslam S, Alavoine G, Benoit P, Garnier P, Recous S. 2015. Rain regime and soil type affect the $\mathrm{C}$ and $\mathrm{N}$ dynamics in soil columns that are covered with mixed-species mulches. Plant Soil 393: 319-334.

MAAF. 2015. Contribution de l'agriculture à la lutte contre le changement climatique : Stéphane Le Foll annonce le lancement d'un projet de recherche international : le « 4 pour 1000 ». Communiqué de presse du 17 mars. Disponible sur http:// agriculture.gouv.fr/contribution-de-lagriculture-la-lutte-contre-lechangement-climatique-lancement-dun-projet-de (dernière consultation le 14/04/2016).

Maltas A, Corbeels M, Scopel E, Wery J, da Silva FAM. 2009. Cover crop and nitrogen effects on maize productivity in notillage systems of the Brazilian cerrados. Agron. J. 101(5): 1036-1046.

Naudin K, Scopel E, Andriamandroso ALH, et al. 2011. Trade-offs between biomass use and soil cover. The case of rice-based cropping systems in the lake Alaotra region of Madagascar. Exp. Agric. 48(2): 194-209.

Neto MS, Scopel E, Coorbeels M, et al. 2010. Soil carbon stocks under no-tillage mulch based cropping systems in the Brazilian cerrado: an on-farm synchronic assessment. Soil Tillage Res. 110(1): 187-195.

Oorst K, Merckx R, Grehan E, Labreuche J, Nicolardot B. 2007. Determinants of annual fluxes of CO2 and N2O in long-term notillage and conventional tillage systems in northern France. Soil Tillage Res. 95: 133-148.

Pascault N, Nicolardot B, Bastian F, Thiébeau P, Ranjard L, Maron PA. 2010. In situ dynamics and spatial heterogeneity of soil bacterial communities under different crop residue management. Environ. Microbiol. 60:291-303.

Ringselle B, Bergkvist G, Aronsson H, Andersson L. 2014. Undergrown-sown cover and post-harvest mowing as measures to control Elymus repens. Eur. Weed Res. Soc. 55: 309-319.

Seguin B, Soussana J-F. 2006. Le réchauffement climatique (prédictions futures et observations récentes) en lien avec les émissions de GES. Fourrages 186: 139-154.

Sinclair TH, Muchow RC. 1999. Radiation use efficiency. Adv. Agron. 65: 215-265.

Systat Software. 2010. SigmaPolt 12. Disponible sur https:// systatsoftware.com/products/sigmaplot/sigmaplot-details.php (dernière consultation le 14/04/2016).

Citation de l'article: Thiebeau P, Recous S. 2016. Une méthode pour quantifier les biomasses de résidus de récolte à la surface des sols après la moisson. Cah. Agric. 25: 45001. 